\title{
Effect of ulinastatin combined with mild therapeutic hypothermia on intestinal barrier dysfunction following cardiopulmonary resuscitation in rats
}

\author{
FANG-JIE ZHANG ${ }^{1}$, HUA-QIANG SONG ${ }^{2}$ and XIANG-MIN LI ${ }^{1}$ \\ ${ }^{1}$ Department of Emergency Medicine, Xiangya Hospital of Central South University, Changsha, Hunan 410008; \\ ${ }^{2}$ Department of Emergency Medicine, The First People's Hospital of Changde City, Changde, Hunan 415000, P.R. China
}

Received January 17, 2019; Accepted August 21, 2019

DOI: $10.3892 /$ etm.2019.8039

\begin{abstract}
The aim of the present study was to investigate the effect of ulinastatin (UTI) alone or combined with mild therapeutic hypothermia (MTH) on intestinal barrier dysfunction following cardiopulmonary resuscitation (CPR) in rats. A total of 25 adult male Sprague-Dawley rats were randomly organized into five groups: Sham; control; UTI; MTH; and the combined group. The latter four groups were induced with the asphyxiated cardiac arrest rat model and treated with different interventions. After $6 \mathrm{~h}$ of treatment, the intestinal tissues of the rats were examined by electron microscopy, and the levels of intestinal malondialdehyde (MDA) and superoxide dismutase (SOD) were determined. The results of the present study indicated that the target temperature had successfully been attained in MTH and the combined group, and the other three groups of rats all survived at a normal temperature. In the rats treated with UTI or MTH, the epithelial cells exhibited pathological changes in their tight junctions and epithelial cell surface microvilli compared with the sham group. In the rats treated with a combination of UTI and MTH, whilst the epithelial cells exhibited a few slight changes, including mitochondrial edema, they were largely similar to the normal epithelial cells. However, there were significant differences in the levels of MDA and SOD between the different treatment groups. UTI combined with MTH may serve a protective role by suppressing oxidative stress in the small intestinal mucosa following CPR in rats compared with either UTI or MTH treatment alone.
\end{abstract}

Correspondence to: Professor Xiang-Min Li, Department of Emergency Medicine, Xiangya Hospital of Central South University, 87 Xiangya Road, Changsha, Hunan 410008, P.R. China E-mail:276856610@qq.com

Key words: cardiopulmonary resuscitation, ulinastatin, mild therapeutic hypothermia, ischemia/reperfusion, intestinal barrier

\section{Introduction}

Cardiac arrest (CA) is a leading cause of mortality that affects $>325,000$ people in the United States of America each year. Sudden cardiac arrest may lead to a loss of blood supply, ischemia and hypoxia in various organs of the body, causing serious damage to organ function and potentially mortality $(1,2)$. Although knowledge and proficiency of cardiopulmonary resuscitation (CPR) have expanded amongst the general population, the rate of survival to hospital discharge following pulseless cardiac arrest remains low (2). However, although resumption of spontaneous circulation (ROSC) following prolonged and complete, whole-body ischemia is an unnatural pathophysiological state created by CPR, in a recent study of 24,132 patients in the United Kingdom who were admitted to critical care units following a cardiac arrest, the in-hospital mortality rate was $71 \%$ (3).

High rates of morbidity and mortality are observed in patients in which ROSC occurs following out-of-hospital CA, which is largely due to the cerebral and cardiac dysfunction that accompanies prolonged whole-body ischemia $(4,5)$, referred to as post cardiac arrest syndrome (PACS). PACS is comprised of anoxic brain injury, post-cardiac arrest myocardial dysfunction, systemic ischemia/reperfusion (I/R) response and persistent precipitating pathology (6). The intestine is equally or potentially even more sensitive to ischemia compared with the brain or muscles following CPR (7). CA causes a prolonged decrease of blood flow to the intestine (7). The $\mathrm{I} / \mathrm{R}$ response in the intestine increases intestinal barrier permeability and results in the translocation of pathogenic bacteria and endotoxins, which is considered as an important mechanism leading to the initiation of sepsis and multiple organ failure $(8,9)$.

Mild therapeutic hypothermia (MTH), also referred to as targeted temperature management, was introduced into the clinical management of cardiac arrest survivors $>10$ years ago $(10,11)$. Therapeutic hypothermia $(\mathrm{TH})$ is now recommended in international resuscitation guidelines, and its use has been extended to cardiac arrest of other origins, with other dysrhythmias and in the hospital setting $(12,13)$. Previous studies have confirmed that $\mathrm{TH}$ and postconditioning with sevoflurane affected the expression and activity of several 
small intestinal proteins that may be involved in intestinal I/R-mediated events following successful CPR $(14,15)$.

Ulinastatin (UTI), a urinary trypsin inhibitor, is a serine protease inhibitor which has anti-inflammatory properties at sites of inflammation and has been widely used as a treatment for patients with inflammatory disorders. UTI has been demonstrated to represent an attractive 'rescue' therapeutic option for endotoxin-associated inflammatory disorders including disseminated intravascular coagulation, acute lung injury and acute liver injury (16-18). UTI treatment at the onset of CPR also alleviates the inflammatory responses following resuscitation and improves neurological function $(19,20)$. In a meta-analysis, UTI decreased the plasma levels of pro-inflammatory cytokines and increased the levels of anti-inflammatory cytokines in patients from China and Japan undergoing cardiac surgery with cardiopulmonary bypass. UTI treatment may have protective effects on myocardial injury and may increase the frequency of auto resuscitation, and shorten the duration of intubation and mechanical ventilation (21).

However, the effects of UTI combined with MTH on intestinal barrier dysfunction in vivo have yet to be determined. The aim of the present study was to investigate the effect of UTI alone or combined with MTH on intestinal barrier dysfunction following cardiopulmonary resuscitation in rats.

\section{Materials and methods}

Animals and chemicals. The experiments performed in the present study were approved by The Animal Research Committee of Xiangya Hospital of Central South University (Changsha, China), and the animals were housed and subsequently used in the Experimental Animal Center of the Xiangya Hospital of Central South University (Changsha, China). A total of 25 adult male Sprague-Dawley rats $(400 \pm 40 \mathrm{~g})$ were purchased from Shanghai Laboratory Animal Center, Chinese Academy of Science. During the entire experiment the animals were housed in stainless steel cages ( 1 rat/cage) at conventional controlled conditions (temperature $25 \pm 2{ }^{\circ} \mathrm{C}$; relative humidity $50 \pm 10 \%$; 12-h light: dark cycle) and had ad libitum access to standard laboratory food and tap water. The rats were randomly assigned to five groups of 5 rats each. The first group was the sham group, in which anesthesia, endotracheal intubation and insertion of arterial and venous catheters were performed, and asphyxiated CA was not induced. Rats in the other four groups, including the control group, the UTI group, the MTH group and the combined group (UTI combined with MTH) were established based on the rat model of CPR following asphyxiated CA. Rats in these four groups underwent the following interventions: Anesthesia; endotracheal intubation; and insertion of arterial and venous catheters; and asphyxiated CA. The UTI group received $1.5 \mathrm{mg} / 100 \mathrm{~g}$ UTI (Guangdong Techpool Bio-pharma, Co., Ltd.) at the onset of resuscitation within the first $2 \mathrm{~min}$, whereas the MTH group were cooled over $5 \mathrm{~min}$ through the application of $70 \%$ isopropyl alcohol to their ventral surface followed by hand fanning after $2 \mathrm{~min}$ of ROSC to achieve a target rectal temperature $\left(32.5 \pm 0.5^{\circ} \mathrm{C}\right)$ in $30 \mathrm{~min}$. On reaching the target temperature of $32.5 \pm 0.5^{\circ} \mathrm{C}$, it was maintained with a cooling blanket [model no, P\&C-A II; Heng Bang (Beijing) Technology Development Co., Ltd.] for an additional $6 \mathrm{~h}$. For rats not in the MTH or UTI and MTH groups, the rectal temperature was maintained at $37^{\circ} \mathrm{C}$ for an additional $6 \mathrm{~h}$.

Establishment of a rat model of CPR following asphyxiated CA. The rats acclimated to conditions for 1 week prior to the experiment and fasted overnight prior to surgery, with ad libitum access to water. The asphyxiated CA rat model was induced as previously described (22). Briefly, rats were anesthetized with $10 \%$ chloral hydrate $0.3 \mathrm{ml} / 100 \mathrm{~g} \mathrm{(300} \mathrm{mg/kg),} \mathrm{orally} \mathrm{intubated}$ and mechanically ventilated using a small-animal ventilator (PE 200 catheter) to maintain an end-tidal $\mathrm{CO}_{2}$ level between 35 and $45 \mathrm{mmHg}$. The rats were intravenously injected with $0.2 \mathrm{mg} / 100 \mathrm{~g}$ vecuronium bromide to prevent spontaneous respiration. The left femoral artery and vein were separately cannulated with PE-50 tubes to measure arterial pressure, heart rate (HR) and arterial blood gas and in order to provide fluid and medications. A standard II lead electrocardiogram was also recorded. Following surgical preparation, the animals were observed for $30 \mathrm{~min}$ to ensure hemodynamic stability. Asphyxiated CA was induced by switching off the ventilator for $30 \mathrm{~min}$. When the electrocardiogram indicated ventricular fibrillation (VF), no pulse electrical activity or electrical static and mean arterial pressure (MAP) below $20 \mathrm{mmHg}$, this was defined as CA (22).

Cardiopulmonary resuscitation was performed on the asphyxiated CA rats after 1 min of CA. CPR was performed with continuous ventilation at a tidal volume of $0.50 \mathrm{ml} / 100 \mathrm{~g}$ with $21 \% \mathrm{O}_{2}$, at a frequency of 100 breaths/min and external cardiac compressions at a rate of $200 / \mathrm{min}$. The compression depth was $1 / 3$ of the anteroposterior diameter of the thorax, along with an intravenous injection of $0.02 \mathrm{mg} / 100 \mathrm{~g}$ epinephrine. If VF persisted or an organized rhythm with a mean aortic pressure $\leq 25 \mathrm{mmHg}$ ensued, defibrillation was attempted after $1 \mathrm{~min}$ of chest compression by delivering up to $2 \mathrm{~J}$ electric shock biphasic waveform electrical shocks across the chest (Heartstream XL; Philips Medical Systems, Inc.). After $30 \mathrm{sec}$ of CPR, 1 3-J monophasic electric shock was administered. Successful CPR was defined as achievement of ROSC, and ROSC was defined as the return of an organized rhythm with a systolic pressure $>80 \mathrm{mmHg}$ for $>5$ min (22). CPR was considered to have failed if no sign of ROSC was observed during the 5 min post-resuscitation period (22).

Assessment of the circulatory function. MAP, HR and rectal temperature were recorded for all the animals during the experimental process.

Histological examination. All rats were re-anesthetized as described above [10\% chloral hydrate $0.3 \mathrm{ml} / 100 \mathrm{~g}(300 \mathrm{mg} / \mathrm{kg})]$ and then sacrificed by cervical spinal cord dissection after $6 \mathrm{~h}$ treatment. No signs of peritonitis were observed. Tissue section samples of the ileum $(1.0 \mathrm{~cm})$ were snap-frozen in liquid nitrogen and stored at $-70^{\circ} \mathrm{C}$ prior to analysis. For analysis, the samples were cut with a cryostat into $2 \times 2 \mathrm{~mm}$ thick sections, fixed in $2.5 \%$ glutaric acid at room temperature for $24 \mathrm{~h}, 2.0 \%$ osmic acid for $2 \mathrm{~h}$ and subsequently dehydrated in a series of 50, 70, 90 and $100 \%$ acetone for 3 cycles, $10 \mathrm{~min} /$ cycle. Following dehydration, all the samples were infused for $24 \mathrm{~h}$ in 1:1 epoxy resin mixture and pure acetone. The samples were 


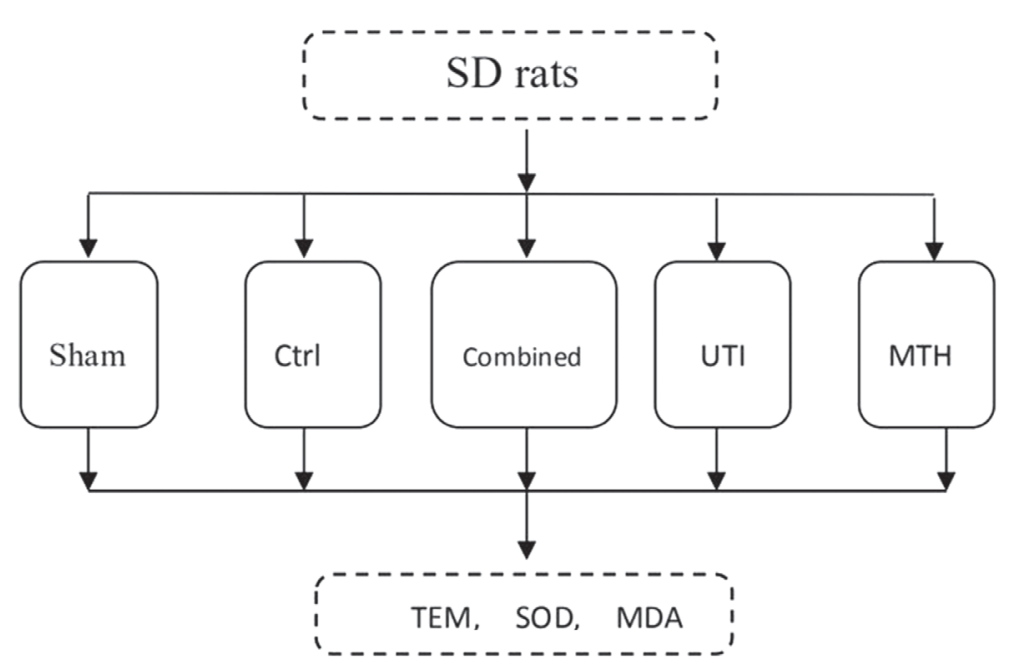

Figure 1. Flow chart of the experimental groups. SD, Sprague-Dawley; Ctrl, control; UTI, ulinastatin; MTH, mild therapeutic hypothermia; TEM, transmission electron microscopy; SOD, superoxide dimutase; MDA, malondialdehyde.

then embedded in ethoxyline resin, dodecenylsuccinic anhydride, methyl nadic anhydride, dimethylaminomethyl phenol at $60^{\circ} \mathrm{C}$ for $24 \mathrm{~h}$. Following embedding, the samples were dyed with $1 \%$ toluidine blue at $50^{\circ} \mathrm{C}$ for $20 \mathrm{~min}$ and cut with an LKB-III ultrathin slicing machine (LKB Ltd.) into $500 \AA$ slices. The slices were then double-stained with $7.7 \%$ uranium acetate for $10 \mathrm{~min}$ and $4 \%$ lead nitrate for $5 \mathrm{~min}$ at room temperature and examined using a HT7700 type transmission electron microscope (Hitachi, Ltd.).

Malondialdehyde (MDA) and superoxide dismutase (SOD) assays in intestinal tissues. Intestinal tissues $(1.0 \mathrm{~cm}$ segments from $5 \mathrm{~cm}$ of the terminal ileum) were harvested, frozen immediately and stored at $-80^{\circ} \mathrm{C}$ until assessment. The levels of MDA in the ileum samples were measured using MDA detection kit (cat. no. A003-1; Nanjing Jiancheng Bioengineering Institute), according to the manufacturer's protocol. Briefly, tissues from the small intestine were homogenized in $1.5 \%$ cold $\mathrm{KCl}$ solution at a ratio of $1: 10$ (weight: Volume). The lipid peroxide level in the supernatant $(1,000 \mathrm{x} \mathrm{g} ; 10 \mathrm{~min}$; room temperature) was measured as described previously (23). Absorbance of the reaction was measured at $532 \mathrm{~nm}$ (Shimadzu UV-1700; Shimadzu Corporation). The lipid peroxide levels are expressed as nmol of MDA/mg of tissue protein. The content of MDA in the intestine was calculated according to the following formula: MDA $(\mathrm{nmol} / \mathrm{mg}$ protein $)=\{[$ (sample tube absorbance-blank tube absorbance)/(standard tube absorbance-blank tube absorbance)] x standard concentration\}/protein content.

Intestinal SOD activity was determined using Superoxide Dismutase assay kit (cat. no. A001-3) according to the manufacturer's protocol (Nanjing Jiancheng Bioengineering Institute). The tissue homogenate and reagent were placed in a water bath at $37^{\circ} \mathrm{C}$ for $40 \mathrm{~min}$ following thorough mixing. After $10 \mathrm{~min}$ at room temperature, the absorbance value of each supernatant was determined at $550 \mathrm{~nm}$. The activity of SOD in the intestine was calculated according to the following formula: SOD $(\mathrm{U} / \mathrm{ml})=[$ control tube absorbance-determination tube absorbance)/control tube absorbance $] /(50 \% \mathrm{x}$ reaction system dilution multiple $\mathrm{x}$ sample dilution multiple).
Statistical analysis. All results statistics were analyzed by GraphPad Prism v.5 (GraphPad Software, Inc.) for statistical evaluation. Data are expressed as the mean \pm standard error of the mean. The statistical analysis of differences between experimental groups was performed using a Student's t-test. A one-way analysis of variance followed by Student-Newman-Keuls test was used to analyze the differences among three experimental groups. $\mathrm{P}<0.05$ was considered to indicate a statistically significant difference.

\section{Results}

Changes in physiological parameters in rats from each group. MAP, HR and rectal temperature of each group were measured prior to euthanizing the rats following the various treatments for $6 \mathrm{~h}$ after ROSC (Fig. 1). MAP and HR values of each group were not significantly different (Fig. 2A and B). The MTH group and combined group rats successfully attained the target temperature, and the other three groups all survived at a normal temperature (Fig. 2C).

Histological changes. The epithelial cells of the small intestinal mucosa were altered following different treatments for 6 h (Fig. 3). Fig. 3A demonstrates that the sham group had the normal epithelial cells, whereas the epithelial cells in the rats in the combined group exhibited indications of slight changes including mitochondrial edema (Fig. 3C). The epithelial cells in the UTI and the MTH groups demonstrated more marked pathological changes, including shortened and partially opened intercellular tight junctions and disorderly epithelial cell surface microvilli (Fig. 3D and E). The control group exhibited the most notable changes to the epithelial cells, including disappearance of the intercellular space and broken microvilli, and the nucleus had indications of chromatin concentration and edge accumulation (Fig. 3B).

MDA and SOD expression assays in intestinal tissue samples. After the $6 \mathrm{~h}$ treatment steps, the levels of MDA in the intestine of the sham, combined, UTI and MTH groups were decreased when compared with the control group (all $\mathrm{P}<0.05$; Table I; Fig. 4A). 

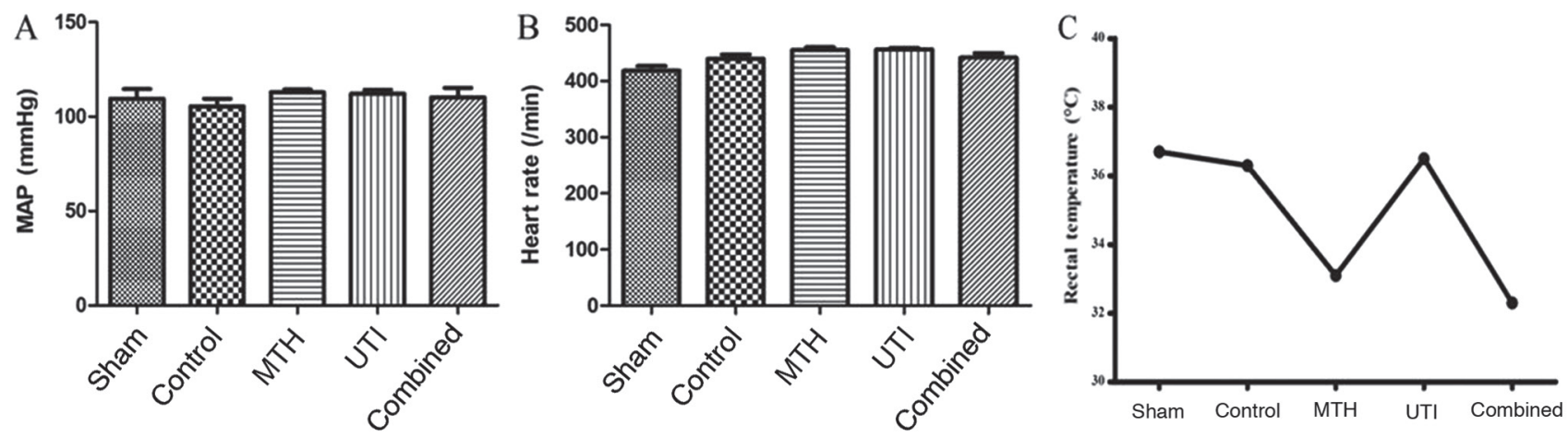

Figure 2. Changes in physiological parameters in the rats from each experimental group. (A) MAP of each treatment group following ROSC. There were no significant differences between the groups $(\mathrm{P}>0.05)$. (B) Heart rate of each group following ROSC. There were no significant differences between the groups $(\mathrm{P}>0.05)$. (C) The MTH group and the combined group rats successfully achieved mild hypothermia, and the rats in the other three groups maintained their normal rectal temperature. MAP, mean arterial pressure; ROSC, resumption of spontaneous circulation; MTH, mild therapeutic hypothermia; UTI, ulinastatin.
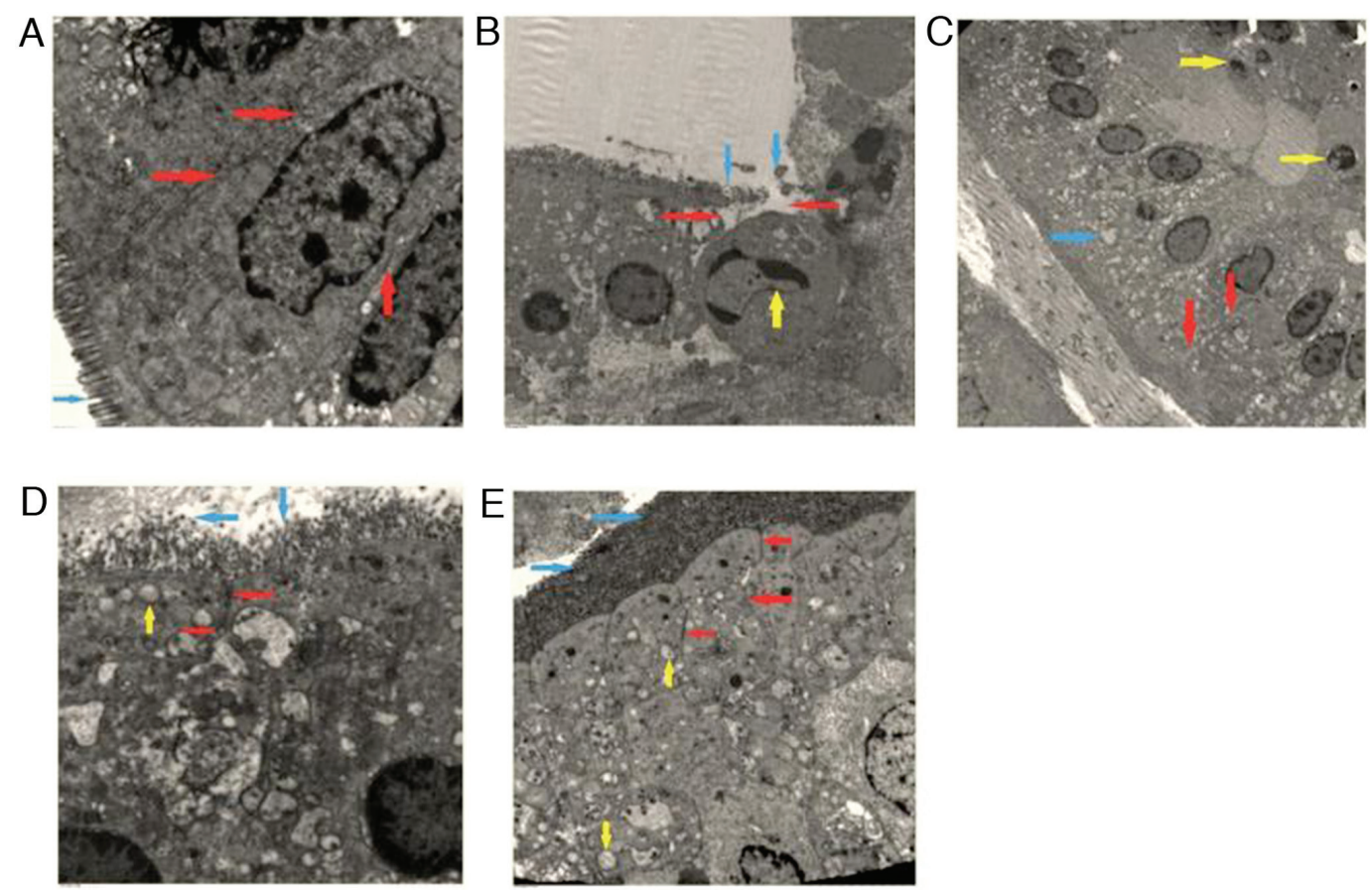

Figure 3. Histological changes in the intestinal tissues isolated from rats in each experimental group. (A) Sham group. Intestinal epithelial cells were arranged neatly (blue arrow), and the epithelial cells were tightly connected and dense (red arrow). (B) Control group. The tight junctions between the epithelial cells disappeared (red arrow), the microvilli of the epithelial cells exhibited necrosis (blue arrow) and the nucleus of the epithelial cells had contracted (yellow arrow). (C) Combined group. Lymphocytes infiltrated under the small intestine epithelium (yellow arrow), tight junctions between epithelial cells were tightly connected (red arrow), and there was evidence of mitochondrial edema (blue arrow). (D) Ulinastatin group. Mitochondrial edema (yellow arrow) was observed and tight junctions between epithelial cells were partially open (red arrow). The microvilli of the epithelial cells were arranged in a disorderly manner and varied in height (blue arrow). (E) Mild therapeutic hypothermia group. The tight junctions between the epithelial cells were open (red arrow), the microvilli were neatly arranged without necrosis and there was evidence of mitochondrial edema (blue arrow). The nucleus had not condensed (yellow arrow). Magnification, $\mathrm{x} 40,000$.

The MDA levels in the combined, UTI and MTH groups were significantly increased compared with the sham group $(\mathrm{P}<0.05)$ and the MDA levels in UTI and MTH groups were significantly increased compared with the combined group $(\mathrm{P}<0.05)$, but there were no significant difference between the UTI and MTH groups $(\mathrm{P}>0.05)$. The results indicated that the levels of MDA in each group were significantly different, with the exception of the comparison between the UTI and MTH groups.

The levels of SOD in the intestinal tissues of the sham, combined, UTI and MTH groups were significantly increased compared with the control group $(\mathrm{P}<0.05$; Fig. 4B; Table II).
The SOD levels in the UTI and MTH groups were decreased compared with the combined group $(\mathrm{P}<0.05)$ and there was no significant difference between the UTI and MTH groups $(\mathrm{P}>0.05)$. These results suggested that the levels of SOD in each group was statistically significant, with the exception of the comparison between the UTI and MTH groups.

Taken together, the results indicated that when compared with the control group, the sham group exhibited relatively decreased levels of MDA and increased levels of SOD. However, the UTI and MTH groups demonstrated decreased levels of MDA and increased levels of SOD compared with 
Table I. Levels of MDA in each group.

\begin{tabular}{lcc}
\hline Group & Sample & MDA (nmol/mg protein) \\
\hline Sham & 5 & $3.61 \pm 0.06^{\mathrm{a}-\mathrm{d}}$ \\
Control & 5 & $4.99 \pm 0.10^{\mathrm{a}, \mathrm{e}-\mathrm{g}}$ \\
UTI & 5 & $4.39 \pm 0.12^{\mathrm{b}, \mathrm{e}, \mathrm{h}, \mathrm{i}}$ \\
MTH & 5 & $4.43 \pm 0.09^{\mathrm{c}, \mathrm{f}, \mathrm{h}}$ \\
Combined & 5 & $3.79 \pm 0.14^{\mathrm{d}, \mathrm{g}, \mathrm{i}}$ \\
\hline
\end{tabular}

Data are presented as the mean \pm standard error of the mean. ${ }^{\mathrm{a}} \mathrm{P}<0.05$, Sham vs. Control; ${ }^{b} \mathrm{P}<0.05$, Sham vs. Combined; ${ }^{\mathrm{c}} \mathrm{P}<0.05$, Sham vs. UTI; and ${ }^{\mathrm{d}} \mathrm{P}<0.05$, and Sham vs. MTH. ${ }^{\mathrm{e}} \mathrm{P}<0.05$, Control vs. Combined; ${ }^{\mathrm{f}} \mathrm{P}<0.05$, Control vs. UTI; ${ }^{\mathrm{P}} \mathrm{P}<0.05$, Control vs. MTH; ${ }^{\mathrm{h}} \mathrm{P}<0.05$, Combined vs. UTI; and $\mathrm{P}<0.05$, Combined vs. MTH. MDA, malondialdehyde; SOD, superoxide dismutase; UTI, ulinastatin; MTH, mild therapeutic hypothermia.

A Expression of MDA

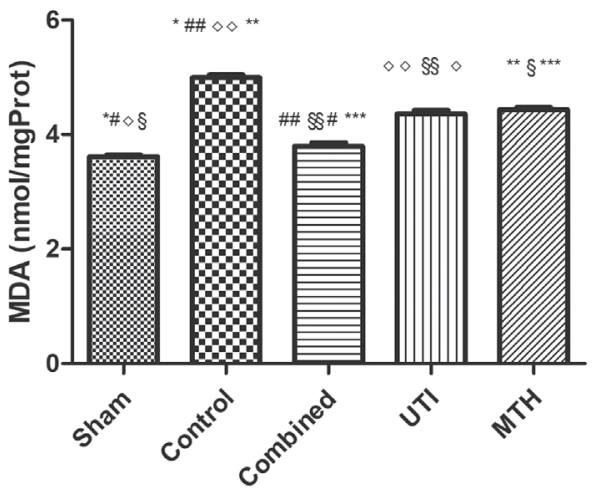

B Expression of SOD

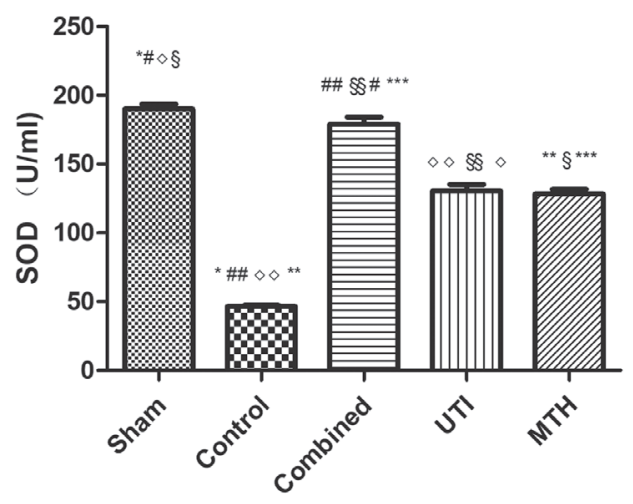

Figure 4.MDA and SOD expression measurements in intestinal tissue samples from rats from each experimental group. (A) Expression of MDA in each group. There was no significant difference between the UTI and MTH groups $(\mathrm{P}>0.05)$. (B) Expression of SOD in each group. There was no significant difference between the UTI and MTH groups $(\mathrm{P}>0.05)$. ${ }^{\mathrm{P}} \mathrm{P}<0.05$, Sham vs. Control; ${ }^{*} \mathrm{P}<0.05$, Sham vs. Combined; ${ }^{\triangleright} \mathrm{P}<0.05$, Sham vs. UTI; and ${ }^{\circledR} \mathrm{P}<0.05$, and Sham vs. MTH. ${ }^{\# \#} \mathrm{P}<0.05$, Control vs. Combined; ${ }^{\diamond} \mathrm{P}<0.05$, Control vs. UTI; ${ }^{* *} \mathrm{P}<0.05$, Control vs. MTH; ${ }^{\S} \mathrm{P}<0.05$, Combined vs. UTI; and ${ }^{* * *} \mathrm{P}<0.05$, Combined vs. MTH. MDA, malondialdehyde; SOD, superoxide dismutase; UTI, ulinastatin; MTH, mild therapeutic hypothermia.

the sham group, but there was no difference between them. The combined group exhibited the lowest levels of MDA and the highest levels of SOD.
Table II. Levels of SOD in the intestinal tissues of each group.

\begin{tabular}{lcc}
\hline Group & Samples & SOD $(\mathrm{U} / \mathrm{ml})$ \\
\hline Sham & 5 & $46.46 \pm 1.88^{\mathrm{a}-\mathrm{d}}$ \\
Control & 5 & $190.20 \pm 7.68^{\mathrm{a}, \mathrm{e}-\mathrm{g}}$ \\
UTI & 5 & $130.61 \pm 10.426^{\mathrm{b}, \mathrm{e}, \mathrm{h}, \mathrm{i}}$ \\
MTH & 5 & $128.53 \pm 5.836^{\mathrm{c}, \mathrm{f}, \mathrm{h}}$ \\
Combined & 5 & $179.15 \pm 11.17^{\mathrm{d}, \mathrm{g}, \mathrm{i}}$ \\
\hline
\end{tabular}

Data are presented as the mean \pm standard error of the mean. ${ }^{\mathrm{a}} \mathrm{P}<0.05$, Sham vs. Control; ${ }^{\mathrm{b}} \mathrm{P}<0.05$, Sham vs. Combined; ${ }^{\mathrm{c}} \mathrm{P}<0.05$, Sham vs. UTI; and ${ }^{\mathrm{d}} \mathrm{P}<0.05$, and Sham vs. MTH. ${ }^{\mathrm{e}} \mathrm{P}<0.05$, Control vs. Combined; ${ }^{\mathrm{f}} \mathrm{P}<0.05$, Control vs. UTI; ${ }^{g} \mathrm{P}<0.05$, Control vs. MTH; ${ }^{\mathrm{h}} \mathrm{P}<0.05$, Combined vs. UTI; and ${ }^{\mathrm{P}}<0.05$, Combined vs. MTH. SOD, superoxide dismutase; UTI, ulinastatin; MTH, mild therapeutic hypothermia.

\section{Discussion}

Intestinal ischemia is one of the major causes leading to multiple organ failure in patients in intensive care facilities and has been described as a consequence of low splanchnic blood flow following cardiac arrest (7). The intestine is not only a vulnerable target to ischemia but a potential secondary source of inflammatory cytokines. Clinically, intestinal injuries following cardiac arrest are often underestimated, even though they represent a potentially devastating complication following successful resuscitation (24). Loss of intestinal barrier integrity serves a pivotal role in the development of systemic inflammatory response syndrome, sepsis and multiple organ failure. The formation of the intestinal mucosal barrier may be associated with the peculiar anatomical-physiological arrangements of the intestinal villi and the epithelial cells (23). Intestinal mucosal surfaces are lined by epithelial cells. These cells establish a barrier between occasionally hostile external environments and the internal milieu. The intestinal epithelial cells serve as a central mediator of interactions between the mucosal immune system and luminal contents, including dietary antigens and microbial products (25). Tight junctions seal the paracellular space between epithelial cells, are the rate-limiting step in transepithelial transport and are the principal determinant of mucosal permeability to regulate the absorption of nutrients (25). Therefore, the epithelium and tight junctions are modulators of mucosal homeostasis.

Previous studies have suggested that UTI may attenuate multiple organ injury in a cardiac arrest model by suppressing inflammation, central autophagy and apoptosis, and antioxidative mechanisms $(20,26,27)$. The present study demonstrated that in the in the control group, the epithelial cells demonstrated the most notable pathological changes; although the rats in this group achieved ROSC following CA, they did not receive any effective intervention and the small intestinal mucosa endured an unnatural pathophysiological ischemic state as a result of CA, which caused a prolonged decrease of blood flow to the intestinal wall (7). Even when treated with UTI or MTH, the epithelial cells in rats demonstrated pathophysiological changes, including alterations to the tight 
junctions and epithelial cell surface microvilli when compared with the sham group. These changes suggested that UTI or MTH only had a partly protective effect on the small intestinal mucosa barrier. The rats that underwent the combined treatment had epithelial cells that presented with only slight pathophysiological changes, including mitochondrial edema, but in all other respects were highly similar to the sham group. The results of the present study suggest that UTI combined with MTH may significantly decrease I/R injury in the small intestinal mucosa.

The levels of the MDA and SOD in the intestinal tissues were additionally examined in the present study. The results of the MDA and SOD assays corroborated the results of the electron microscopy examination of the ileum tissues. UTI and MTH separately partly protected against injury induced as a result of I/R of the small intestinal mucosa; however, UTI combined with MTH significantly improved intestinal mucosa barrier function in rats that achieved ROSC following CA. MDA is often used as an indicator of oxidative stress, as it is degradation product of polyunsaturated lipids by reactive oxygen species and may cause toxic stress in cells, whereas SOD is important antioxidant in cells exposed to oxygen (8). Therefore, UTI combined with MTH may improve antioxidant defense and suppress the oxidative stress in the small intestinal mucosa.

The intestinal mucosa is an organ with a rich blood supply and extensive metabolic activities, and therefore it is also vulnerable to metabolic disorders caused by hypoxia. In addition, I/R injury often occurs when reperfusion is achieved due to abnormal oxygen metabolism, the generation of oxygen free radicals, calcium overload and endothelial swelling (28). When intestinal injury is caused by reperfusion, the primary characteristics are expression of adhesion molecules, production of inflammatory cytokines, activation of the complement system, infiltration of neutrophil and release of proteases, and UTI may directly inhibit the production of these molecular and cellular factors, or directly affect neutrophils, inhibiting its infiltration into the tissues which would otherwise cause inflammatory damage (29). Previous studies have also confirmed that UTI had a significant effect in protecting the mucosal barrier function particularly against early pathological changes $(30,31)$ and that UTI significantly ameliorated injury to the small intestinal and subsequent bacterial translocation by inhibiting degranulation of mast cells in septic rats (32). In addition, a protective effect of UTI on intestinal injury during the perioperative period of acute superior mesenteric artery ischemia has also been observed (29). Previous studies additionally suggested that UTI significantly decreased the levels of MDA in the plasma of patients following early CPR (26), and the systemic administration of UTI also largely restored SOD in CA models (20). The present study confirmed that UTI treatment alone or combined with MTH may serve a protective role by suppressing the expression of SOD and MDA in rats. Patients are prone to multiple organ dysfunction following CPR, but effective treatments are limited at present. UTI has been widely applied in clinical practice in China, and previous studies have confirmed its protective effects (18-21). The present study may provide novel insights into the development of potential therapeutics for clinical treatment of patients following CPR.
Therapeutic hypothermia is now recommended in the international resuscitation guidelines to treat patients post-CA (12). Hypothermia applied as a rescue therapy for intestinal $\mathrm{I} / \mathrm{R}$ resulted in decreased mortality even following returning to normothermia $(33,34)$. Hypothermic protection during early reperfusion appears to be mediated by several events, including prevention of intestinal and pulmonary neutrophil infiltration, a decrease in oxidative stress in the ileum, and preservation of cardiac and hepatic energy metabolism. Moderate hypothermia may improve outcomes in clinical conditions associated with intestinal I/R $(33,34)$. However, relatively few studies have determined the mechanisms underlying the protective effects of MTH on small intestinal injury. Perioperative hypothermia decreased the activity of the antioxidant enzymes catalase and SOD, decreased glutathione levels and increased lipid peroxidation in the scar tissue of colonic anastomoses in rats (35-37). Hypothermia altered the expression and activity of several small intestinal proteins that may be involved in intestinal I/R-mediated events following successful cardiopulmonary resuscitation (14).

The present study has several limitations: Firstly, only the small intestine changes within $6 \mathrm{~h}$ after CPR in rats were observed, but without real-time dynamic monitoring; secondly, the pharmacological mechanisms of MTH and UTI activity were not examined in further detail, to provide additional understanding of the pathways underlying the protective effects of MTH and UTI; thirdly, although UTI and MTH have been widely used in the clinical treatment of patients following CPR, there are certain differences between animal models and actual clinical situations, and therefore the results of the present study may not accurately reflect the results of any potential clinical application; fourthly, as the levels of MDA content and SOD activity are the primary indicators for evaluating the degree of oxidative stress damage in various tissues, and are considered to reflect the initial stage of oxidative stress and I/R injury changes, no other molecules or biomarkers were analyzed in the present study. However, additional molecules or biomarkers involved in intestinal barrier injury will be examined in subsequent studies.

In conclusion, both UTI and MTH alone demonstrated partial protective effects on intestinal mucosal barrier function; however, UTI combined with MTH exhibited an improved protective effect by suppressing oxidative stress in the small intestinal mucosa following CPR in rats.

\section{Acknowledgments}

Not applicable.

\section{Funding}

The present study was supported by the grants from the National Natural Science Foundation of China (grant no., 81501923) and the Provincial Natural Science Foundation of Hunan (grant no., 2018JJ2639) and the Rui E (Ruiyi) Emergency Medical Research Special Funding Project (grant no. R201907).

\section{Availability of data and materials}

The datasets used and/or analyzed during the current study are available from the corresponding author on reasonable request. 


\section{Authors' contributions}

FJZ wrote the manuscript and analyzed the data. XML designed the experiments. FJZ and HQS established the rat models. HQS analyzed all experimental data. FJZ and XML provided the experiment fees. All authors have read and approved the final manuscript.

\section{Ethics approval and consent to participate}

The experiments performed in the present study were approved by The Animal Research Committee of Xiangya Hospital of Central South University.

\section{Patient consent for publication}

Not applicable.

\section{Competing interests}

The authors declare that they have no competing interests.

\section{References}

1. Nichol G, Thomas E, Callaway CW, Hedges J, Powell JL, Aufderheide TP, Rea T, Lowe R, Brown T, Dreyer J, et al Regional variation in out-of-hospital cardiac arrest incidence and outcome. JAMA 300: 1423-1431, 2008.

2. Zhu A and Zhang J: Meta-analysis of outcomes of the 2005 and 2010 cardiopulmonary resuscitation guidelines for adults with in-hospital cardiac arrest. Am J Emerg Med 34: 1133-1139, 2016.

3. Nolan JP, Laver SR, Welch CA, Harrison DA, Gupta V and Rowan K: Outcome following admission to UK intensive care units after cardiac arrest: A secondary analysis of the ICNARC Case Mix programme database. Anaesthesia 62: 1207-1216, 2007.

4. Stub D, Bernard S, Duffy SJ and Kaye DM: Post cardiac arrest syndrome: A review of therapeutic strategies. Circulation 123: 1428-1435, 2011

5. Wang Z, Ye Z, Huang G, Wang N, Wang E and Guo Q: Sevoflurane Post-conditioning enhanced hippocampal neuron resistance to global cerebral ischemia induced by cardiac arrest in rats through PI3K/Akt survival pathway. Front Cell Neurosci 10: 271, 2016.

6. Nolan JP, Neumar RW, Adrie C, Aibiki M, Berg RA, Bbttiger BW, Callaway C, Clark RS, Geocadin RG, Jauch EC, et al: Post-cardiac arrest syndrome: Epidemiology, pathophysiology, treatment, and prognostication. A scientific statement from the international liaison committee on resuscitation; the american heart association emergency cardiovascular care committee; the council on cardiovascular surgery and anesthesia; the council on cardiopulmonary, perioperative, and critical care; the council on clinical cardiology; the council on stroke. Resuscitation 79: 350-379, 2008.

7. Korth U, Krieter H, Denz C, Janke C, Ellinger K, Bertsch T, Henn $\mathrm{C}$ and Klein J: Intestinal ischaemia during cardiac arrest and resuscitation: Comparative analysis of extracellular metabolites by microdialysis. Resuscitation 58: 209-217, 2003.

8. Pan H, Chen D, Liu B, Xie X, Zhang J and Yang G: Effects of sodium hydrosulfide on intestinal mucosal injury in a rat model of cardiac arrest and cardiopulmonary resuscitation. Life Sci 93: 24-29, 2013.

9. Stallion A, Kou TD, Latifi SQ, Miller KA, Dahms BB, Dudgeon DL and Levine AD: Ischemia/reperfusion: A clinically relevant model of intestinal injury yielding systemic inflammation. J Pediatr Surg 40: 470-477, 2005.

10. Hypothermia after Cardiac Arrest Study Group: Mild therapeutic hypothermia to improve the neurologic outcome after cardiac arrest. N Engl J Med 346: 549-556, 2002.

11. Bernard SA, Gray TW, Buist MD, Jones BM, Silvester W, Gutteridge $\mathrm{G}$ and Smith K: Treatment of comatose survivors of out-of-hospital cardiac arrest with induced hypothermia. N Engl J Med 346: 557-563, 2002.
12. Nolan JP, Hazinski MF, Aickin R, Bhanji F, Billi JE, Callaway CW, Castren M, de Caen AR, Ferrer JM, Finn JC, et al: Part 1: Executive summary: 2015 International consensus on cardiopulmonary resuscitation and emergency cardiovascular care science with treatment recommendations. Resuscitation 95: el-e31, 2015.

13. Feng W, Jin L, Xie Q, Huang L, Jiang Z, Ji Y, Li C, Yang L and Wang D: Eugenol protects the transplanted heart against ischemia/reperfusion injury in rats by inhibiting the inflammatory response and apoptosis. Exp Ther Med 16: 3464-3470, 2018.

14. Albrecht M, Gruenewald M, Zitta K, Zacharowski K, Scholz J, Bein B and Meybohm P: Hypothermia and anesthetic postconditioning influence the expression and activity of small intestinal proteins possibly involved in ischemia/reperfusion-mediated events following cardiopulmonary resuscitation. Resuscitation 83: 113-118, 2012.

15. Yang GS, Zhou XY, An XF, Liu XJ, Zhang YJ and Yu D: mTOR is involved in stroke-induced seizures and the anti-seizure effect of mild hypothermia. Mol Med Rep 17: 5821-5829, 2018.

16. Inoue $\mathrm{K}$ and Takano $\mathrm{H}$ : Urinary trypsin inhibitor as a therapeutic option for endotoxin-related inflammatory disorders. Expert Opin Investig Drugs 19: 513-520, 2010.

17. Karnad DR, Bhadade R, Verma PK, Moulick ND, Daga MK, Chafekar ND and Iyer S: Intravenous administration of ulinastatin (human urinary trypsin inhibitor) in severe sepsis: A multicenter randomized controlled study. Intensive Care Med 40: 830-838, 2014.

18. Yang B, Gao M, Wang K, Jiang Y, Peng Y, Zhang H, Yang M and Xiao X: Intraintestinal administration of ulinastatin protects against sepsis by relieving intestinal damage. J Surg Res 211: 70-78, 2017.

19. Sui B, Li Y and Ma L: Postconditioning improvement effects of ulinastatin on brain injury following cardiopulmonary resuscitation. Exp Ther Med 8: 1301-1307, 2014

20. Jiang XM, Hu JH, Wang LL, Ma C, Wang X and Liu XL: Ulinastatin alleviates neurological deficiencies evoked by transient cerebral ischemia via improving autophagy, Nrf-2-ARE and apoptosis signals in hippocampus. Physiol Res 67: 637-646, 2018.

21. Zhang Y, Zeng Z, Cao Y, Du X and Wan Z: Effect of urinary protease inhibitor (ulinastatin) on cardiopulmonary bypass: A meta-analysis for China and Japan. PLoS One 9: e113973, 2014.

22. Han F, Boller M, Guo W, Merchant RM, Lampe JW, Smith TM and Becker LB: A rodent model of emergency cardiopulmonary bypass resuscitation with different temperatures after sphyxial cardiac arrest. Resuscitation 81: 93-99, 2010.

23. Ghali MG and Marchenko V: Dynamic changes in phrenic motor output following high cervical hemisection in the decerebrate rat. Exp Neurol 271: 379-389, 2015.

24. He W, Liu Y, Geng $\mathrm{H}$ and $\mathrm{Li}$ Y: The regulation effect of ulinastatin on the expression of SSAT2 and AQP4 in myocardial tissue of rats after cardiopulmonary resuscitation. Int J Clin Exp Pathol 8: 10792-10799, 2015.

25. Chiu CJ, McArdle AH, Brown R, Scott HJ and Gurd FN: Intestinal mucosal lesion in low-flow states. I. A morphological, hemodynamic, and metabolic reappraisal. Arch Surg 101: $478-483,1970$.

26. Katsoulis IE, Balanika A, Sakalidou M, Gogoulou I, Stathoulopoulos A and Digalakis MK: Extensive colonic necrosis following cardiac arrest and successful cardiopulmonary resuscitation: Report of a case and literature review. World J Emerg Surg 7: 35, 2012.

27. Turner JR: Intestinal mucosal barrier function in health and disease. Nat Rev Immunol 9: 799-809, 2009.

28. Liu Q, Peng J,Zhou Y, Zeng W, Xiao S, Cheng H, Zhong Z, Liao X, Xiao X, Luo L and Liu X: Clinical observation of ulinastatin combined with CRRT in the treatment of early cardiopulmonary resuscitation. Exp Ther Med 14: 6064-6068, 2017.

29. Liu S, Xu J, Gao Y, Shen P, Xia S, Li Z and Zhang M: Multi-organ protection of ulinastatin in traumatic cardiac arrest model. World J Emerg Surg 13: 51, 2018.

30. Sasaki M and Joh T: Oxidative stress and ischemia-reperfusion injury in gastrointestinal tract and antioxidant, protective agents. J Clin Biochem Nutr 40: 1-12, 2007.

31. Qian ZY, Yang MF, Zuo KQ, Xiao HB, Ding WX and Cheng J: Protective effects of ulinastatin on intestinal injury during the perioperative period of acute superior mesenteric artery ischemia. Eur Rev Med Pharmacol Sci 18: 3726-3732, 2014.

32. Tian YF, Li Y, Zhao Q, Fan LQ, Zhao WJ, Xu BL, Song ZC, Kuang G, Dong ZM and Zhang QF: Effect of ulinastatin on intestinal mucosal barrier function of rats with obstructive jaundice. Nan Fang Yi Ke Da Xue Xue Bao 27: 987-990, 2007 (In Chinese). 
33. Ivary SHA, Jajarmy N, Shahri MK, Shokoohi M, Shoorei H, Ebadi A, Moghimian M and Sigaroodi F: Effect of fish and flaxseed oil supplementation on isoprenaline-induced myocardial infarction in rats: Inhibition of mitochondrial permeability transition pore opening. Crescent J Med Biol Sci 6: 158-163, 2019.

34. Zhang YJ, Li M, Meng M, Feng M and Qin CY: The effect of ulinastatin on the small intestine injury and mast cell degranulation in a rat model of sepsis induced by CLP. Exp Toxicol Pathol 61: 481-490, 2009.

35. Stefanutti G, Pierro A, Parkinson EJ, Smith VV and Eaton S: Moderate hypothermia as a rescue therapy against intestinal ischemia and reperfusion injury in the rat. Crit Care Med 36: 1564-1572, 2008.
36. Yang GS, Zhou XY, An XF, Liu XJ, Zhang YJ and Yu D: Mild hypothermia inhibits the Notch 3 and Notch 4 activation and seizure after stroke in the rat model. Pathol Res Pract 214: 1008-1016, 2018

37. Oliveira JC, Oliveira CH, Oliveira HE, Pereira A, Maraschin M and D'Acampora AJ: Effects of perioperative hypothermia and reactive oxygen species in the healing of colonic anastomosis in rats. Acta Cir Bras 29: 742-747, 2014.

(i) (2) This work is licensed under a Creative Commons Attribution-NonCommercial-NoDerivatives 4.0 International (CC BY-NC-ND 4.0) License. 